\title{
Pour une biologie moléculaire darwinienne
}

Pour la biologie moderne, l'origine et le fonctionnement du vivant sont attribués à l'ADN. Le programme génétique a tout prévu pour notre plus grand bénéfice et en détient les clés: embryogenèse, fonctions, organes, régulations... Pour les premiers naturalistes, les grands équilibres naturels relevaient d'une conception analogue: le programme, celui de la création cette fois, expliquait l'origine et le fonctionnement de la nature, conçue de la même manière pour le plus grand bénéfice de l'homme. La biologie moderne ne commet-elle pas, à l'intérieur de l'organisme, l'erreur que les Anciens commettaient à l'extérieur? [1].

Qu'est ce qu'un gène? [2]*. Selon la définition initiale, le gène est une «abstraction » représentant le caractère héréditaire. Associé aux lois de Mendel, le gène permet de faire des «calculs » sur la transmission. Ensuite, les travaux de T. Morgan montrèrent que des locus chromosomiques peuvent être associés aux caractères [3]. L'association n'implique pas la causalité, mais permet aux marqueurs génétiques, version moderne des locus, d'être exploitables pour le diagnostic ou l'étude des populations. La voie était ouverte pour conférer une réalité matérielle et une valeur causale au gène abstrait des mendéliens. On aboutit au « déterminant » génétique de la biologie moléculaire: un segment d'ADN, plus ou moins facile à délimiter, qui code pour la synthèse d'une protéine. Malheureusement, sauf à assimiler le caractère héréditaire à une protéine, il est impossible de confondre le gène-ADN avec le gène-caractère. Le chemin est bien long et tortueux des protéines aux caractères héréditaires et ne dépend pas seulement des facteurs génétiques (le prion en est l'illustration extrême). Les diverses définitions du gène, tout à fait opérationnelles en leur champ propre (généalogie, diagnostic, synthèse des protéines...), ne sont pas cohérentes entre elles.

Cette incohérence accroît la difficulté d'unir les concepts de la génétique à ceux de la théorie darwinienne de

* Sur les difficultés de la définition du gène, voir [2].
Génétique des virus, Institut Cochin,

I'évolution, ce qu'a tenté 22, rue Méchain, Inserm la théorie synthétique (ou néodarwinisme). Selon cette théorie, la sélection U.567, Cnrs UMR 8104, 75014 Paris, France. sonigo@cochin.inserm.fr notypes issus des variations aléatoires du génotype. Le génotype est donc sélectionné « indirectement » par l'intermédiaire du phénotype associé [4]. La théorie synthétique peut être décrite par le « gène égoïste » de R. Dawkins: c'est le gène qui est l'objet de la sélection naturelle, associé à ses « extensions » phénotypiques [5]. Le problème principal de la théorie synthétique, c'est que le chemin qui mène du génotype au phénotype est parcouru avant que la sélection ne puisse opérer. Or, c'est précisément ce chemin qui préoccupe la biologie « post-génomique ». Avant d'arriver au phénotype, la vie échapperait-elle à la sélection naturelle?

Il est possible de résoudre cette difficulté en s'appuyant sur la théorie de l'évolution « à tous les niveaux ». La sélection - correspondant par exemple à des avantages de stabilité, de croissance ou de reproduction - opérant à un niveau arbitrairement choisi produit une organisation qui peut être sélectionnée aux autres niveaux arbitrairement choisis. Schématiquement, la compétition entre les réactions chimiques [6]** produit une organisation - la cellule - qui entre en compétition avec les autres cellules, ce qui définit l'organisme, qui entre en compétition avec les autres organismes, etc. Pour illustrer une telle «fractale darwinienne », la métaphore de l'écosystème peut être utile: l'organisme est une forêt, habitée par des animaux libres et autonomes. Dans un écosystème, il n'y a pas de programme représentant l'objectif collectif des animaux et des plantes, et garantissant la sauvegarde de l'ensemble. La structure globale émerge des interactions individuelles. On peut aussi se référer à la ruche, construite par des abeilles qui n'ont aucun plan ni vision d'ensemble et obéissent à des règles

\footnotetext{
** Plus techniquement, cela correspond aux modèles d'auto-organisation de la
} chimie. Voir par exemple [6]. 
purement locales. De manière analogue, nos cellules s'auto-organiseraient autour de « chaînes alimentaires » fondées sur des avantages métaboliques locaux. Le globule blanc ne serait pas dévoué à la survie de sa collectivité. II dévorerait les microbes parce qu'ils constituent la «nourriture » la plus accessible là où il se trouve. La cellule du foie est voisine de l'intestin. Elle se spécialiserait pour exploiter les ressources qui lui arrivent du tube digestif, non pour assurer que nous aurons bien un foie. La vie est une « conjonction d'intérêts »: l'organisme bénéficie certes de ces spécialisations cellulaires et les re-sélectionne à son niveau. Mais, le finalisme insidieux du programme génétique est évacué: la logique de l'organisme n'explique pas la mise en place des organes. Conséquence troublante dans ce cadre: I'ADN n'est plus le vecteur privilégié de l'hérédité. II n'intervient pas plus que les autres composants qu'ils soient « internes » (nucléaires, cytoplasmiques,...) ou «externes » (locaux, environnementaux,...). Les facteurs externes portent donc l'hérédité tout autant que l'ADN.

Envisageons quelques perspectives ou ouvertures expérimentales.

La thérapie génique ne serait plus conçue comme une « reprogrammation », mais comme une introduction d'espèce dans un écosystème: en fonction des avantages sélectifs, la nouvelle espèce peut s'installer de manière stable et permettre un nouvel état de l'ensemble [7]. L'espèce introduite peut aussi s'éteindre, c'est le cas le plus fréquent en thérapie génique, ou au contraire proliférer et/ou provoquer un déséquilibre mortel. En écologie, la problématique de l'introduction ou de la préservation d'espèce dans un écosystème ne se résume pas à l'efficacité des vecteurs de transfert.

Le cancer ne serait plus conçu comme une violation du traité anti-prolifération imposé par la toute-puissance génétique. Il serait un état de moindre spécialisation tissulaire répondant à une nouvelle distribution des ressources dans l'organisme. Les relations entre le niveau de spécialisation des individus et le partage des ressources disponibles sont une problématique de l'écologie évolutionniste (voir par exemple [8]). II serait passionnant d'importer ces outils et concepts et d'étudier sous cet angle les microenvironnements des cellules normales ou cancéreuses.

Le soi immunologique ne serait plus une catégorie particulière de structures, issues d'un apprentissage. II correspondrait à un état stationnaire de la production et de la consommation des constituants tissulaires. La logique de l'auto-immunité serait recherchée au niveau des dynamiques de consommation des cellules entre

Texte présenté au $18^{\mathrm{e}}$ séminaire de génétique clinique organisé par Arrnold Munnich et Stanislas Lyonnet (Paris, mars 2002). elles, plutôt que dans les anomalies de la régulation postulée des défenses de l'organisme.

La plasticité des cellules souches traduirait leur «adaptabilité » à des ressources environnementales diverses. Selon la théorie de l'évolution, le potentiel adaptatif résulte du taux de variation et de la taille de la population considérée. Inutile de chercher une propriété intrinsèque ou un marqueur fixe de cellule souche si c'est la vitesse de variation et la taille de la population qu'il faut mesurer.

Les grandes fonctions indispensables à notre existence (respiration, digestion, reproduction, etc.) résultent des interactions moléculaires et cellulaires, sans en être la cause. Les molécules et les cellules sont libres. L'individu n'est pas au centre de son monde intérieur. Pour comprendre notre existence, il faut commencer par l'oublier. $\diamond$

In favour of a Darwinian molecular biology

\section{RÉFÉRENCES}

1. Kupiec JJ, Sonigo P. Ni Dieu ni gène, pour une autre théorie de l'hérédité. Collection Science ouverte. Paris: Seuil, 2000.

2. Dossier: Qu'est ce qu'un gène? La Recherche 2001; $n^{\circ}$ 348: 50-60.

3. Pichot A. Histoire de la notion de gène. Paris: Champs Flammarion, 1999.

4. Maynard-Smith J. La construction du vivant. gènes, embryons et évolution. Collection Le sel et le fer. Paris: Cassini, 2001.

5. Dawkins R. The extended phenotype. The long reach of the gene. New York: Oxford University Press, 1982, 1999.

6. Pacault A, Perraud JJ. Rythmes et formes en chimie. Collection Que sais-je. Paris: PUF, 1997.

7. Fischer A, Hacein-Bey S, Le Deist F, Cavazzana-Calvo M. Traitement du déficit immunitaire combiné sévère lié à l'X par transfert ex vivo du gène gc. Med Sci 2000; 16: 681-4.

8. Seligmann H. Resource partition history and evolutionary specialization of subunits in complex systems. Biosystems 1999; 51: 31-9. 